\section{IN THE NEWS}

From mouse to man With the publication of the draft sequence of the C57BL/6J mouse genome in the 5 December issue of Nature, a powerful new resource has become available to the cancerresearch community. "The entire biomedical research community can for the first time fully use this resource to tackle human diseases", said Dr Jane Rogers of the Sanger Institute, UK (bbc.co.uk)

The international sequencing consortium estimates that the 2.5 billion nucleotide genome contains 30,000 genes $99 \%$ of which have a human homologue - this is the first time that the genomes of two mammals have been available for comparison (covered in the New York Times, 5 Dec 2002). Some $96 \%$ of the mouse genes lie in regions that are 'syntenic' with human chromosomes. In an accompanying News and Views article (Nature $\mathbf{4 2 0}$ 515-518 (2002)), Mark Boguski says "the conservation of synteny between mouse and human chromosomes will allow effective cross-reference of the location of any genetically mapped traits in the mouse with genes in the orthologous regions of the human genome. This will greatly accelerate the isolate of disease genes".

The genome sequence will also make mice a better model for mutation-based screening assays. In a News and Views article in the January issue of Nature Genetics, Tim O'Brien and Rick Woychik discuss how the genome sequence will also help with the design and generation of targeted mutations produced by homologous recombination.

The sequencing effort has also led to the discovery of about 1,200 new genes that have human homologues, many of which are likely to have undiscovered cancerrelated functions.

Kris Novak

\title{
The stabilizing influence of VHL
}

Inactivation of the von HippelLindau ( VHL) tumour-suppressor gene is linked to the development of several different tumour types in

humans, including tumours of the kidney, retina, central nervous system and the adrenal gland. Despite identification of the gene almost 10
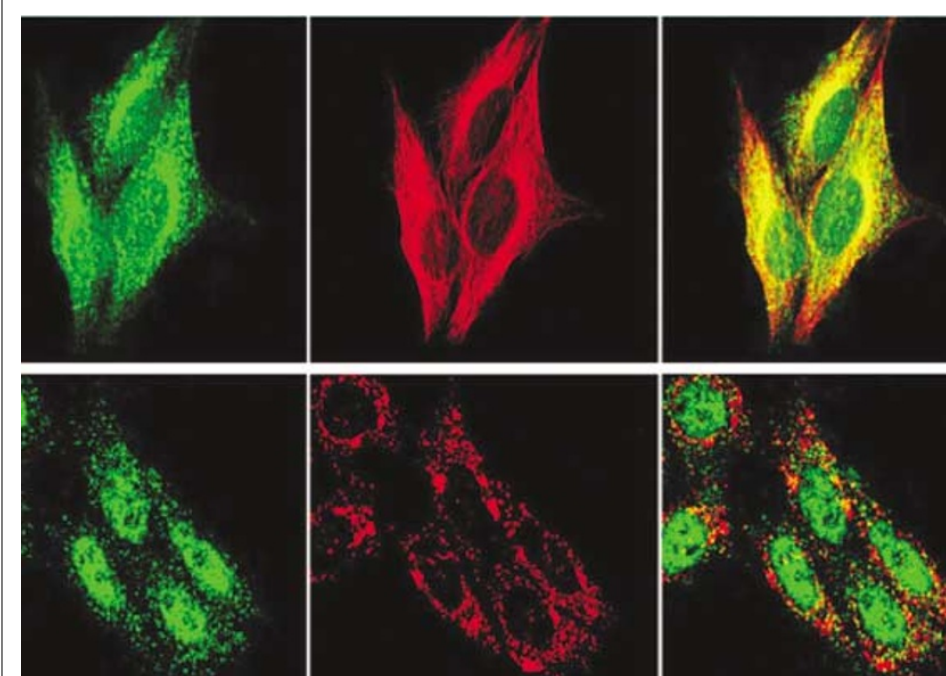

VHL localization depends on an intact microtubule network. $\mathrm{VHL}_{20}$ (green) co-localizes with the microtubule network ( $\beta$-tubulin; red) in HeLa cells (top panel). Co-localization is shown in yellow. This localization of $\mathrm{VHL}_{30}$ is disrupted when microtubules are depolymerized with colcemid (bottom panel).

\section{METASTASIS}

\section{Stay or go?}

At what point do tumour cells acquire their metastatic potential? Are certain primary tumours prone to metastasis, or is the ability to take up residence in a foreign tissue a characterstic of only a few cells that have managed to break free from their primary tumour host? A gene-profiling study, published by Todd Golub and colleagues in the January issue of Nature Genetics, begins to answer these questions.

The authors analysed the geneexpression profiles of 12 metastatic adenocarcinoma nodules from tissues such as lung, breast, prostate, colorectum, uterus and ovary, and compared them with expression profiles of 64 primary adenocarci- nomas representing the same spectrum of tumours. They identified 128 genes that distinguished primary from metastatic adenocarcinomas. Metastasis was associated with the upregulation of a number of genes that regulate protein translation (SNRPF, EIF4EL3, HNRPAB and DHPS). Other upregulated genes seemed to come from the non-epithelial component of the tumour, such as those that encode type I collagens, indicating the importance of the stroma in regulating metastasis. Analysis of additional tumours revealed a similar metastatic gene signature.

This metastasis-associated geneexpression pattern was also present in some primary tumours, so did this mean that these tumours were destined to metastasize? The authors found that patients with primary tumours that expressed the metasta- years ago, we are only now beginning to understand how VHL functions in the cell and how its mutation leads to tumour development. Reporting in the January issue of Nature Cell Biology, Krek and colleagues have now uncovered a novel function for VHL - microtubule stabilization - and show that disruption of this function is linked to the development of a specific subtype of VHL disease.

So far, the best-characterized function of VHL has been as a component of an E3 ubiquitin ligase complex, which mediates degradation of the hypoxia-inducible factor (HIF) under normoxic conditions. Although there are some links between this and particular subtypes of VHL disease, the extent to which this function of VHL contributes to tumour progression is unclear. More recently, VHL has also been implicated in extracellular-matrix formation and cell-cycle progression.

The importance of the new work from Krek and colleagues is that they identify a novel cytoskeletal function, which is specific to an isoform of VHL that has not previously been linked to VHL tumour development.

tic gene profile had significantly shorter survival times than cancer patients whose tumours did not. This means that some primary tumours already have the propensity for metastasis as early as the time of diagnosis.

The authors also looked for the metastasis-associated gene signature in other tumour types, and found that their pattern could be used to predict metastatic potential of small stage I primary breast adenocarcinomas, prostate adenocarcinomas and medulloblastomas. This indicates that there are generic gene-expression programmes associated with the metastatic process in different tumours. Notably, the gene-expression profile was not able to predict metastasis in patients with diffuse large-B-cell lymphoma. This might be because haematopoietic malignancies have special mechanisms for spreading 